\title{
Traduire l'argent : le rôle des compléments cognitifs dans la traduction littéraire
}

\section{Translating Money: The Role of Cognitive Complements in Literary Translation}

\section{Carmen-Ecaterina CIOBÂCĂ ${ }^{1}$ (1)}

'Lecturer, PhD, "Alexandru loan Cuza University", Faculty of Law, Center for European Studies, Iași, Romania

\section{ORCID: C.E.C. 0000-0002-2892-7487}

\section{Corresponding author:}

Carmen-Ecaterina CIOBÂCĂ,

"Alexandru loan Cuza" University, Faculty of Law, Center for European Studies, Iaşi, Romania

E-mail: carmen.ciobaca@gmail.com

Submitted: 31.01 .2021

Revision Requested: 27.07.2021

Last Revision Received: 11.08.2021 Accepted: 27.09.2021

Citation: Ciobaca, C. E. (2021). Traduire I'argent : le rôle des compléments cognitifs dans la traduction littéraire. Litera, 31(2), 835-856.

https://doi.org/10.26650/LITERA2021-871519

\section{RÉSUMÉ}

La présente étude a le but de souligner que, pendant le processus de traduction, le recours aux compléments cognitifs est indispensable. Fondée sur la théorie interprétative de la traduction (TIT), cette approche analyse le traduire comme démarche cognitive. Dans un premier temps, l'auteur examine la manière dont la cognition se manifeste pendant les trois étapes de la traduction : la compréhension, la déverbalisation, la réexpression. Ensuite, l'auteur opère la différence entre le linguistique et l'extralinguistique et présente le rôle des compétences langagières et des connaissances encyclopédiques du traducteur. Les compléments cognitifs, compris comme des éléments d'ordre extralinguistique, notionnels et émotionnels, qui contribuent à la saisie du sens, aident le sujet traduisant à transporter le message non seulement d'une langue à l'autre, mais aussi d'un univers cognitif à l'autre. Confronté à des difficultés de traduction, le traducteur/la traductrice fait appel aux compléments cognitifs et adopte des stratégies appropriées. Pour préserver le spécifique cognitif du texte source, le sujet traduisant recourt à la traduction littérale ou à l'explicitation. Par contre, pour niveler le spécifique de l'univers cognitif évoqué par le texte de départ, le traducteur/la traductrice utilise la traduction généralisante ou l'adaptation. Le travail comporte également une étude de cas : I'analyse de la manière dont ont été traduites en anglais et en roumain des références explicites et implicites à l'argent qui se retrouvent dans un fragment du roman Eugénie Grandet de Balzac. Suite à cette analyse, on observe que les techniques de traduction doivent être choisies en fonction du savoir que le traducteur/la traductrice partage ou non avec le public cible.

Mots-clés: Théorie interprétative de la traduction (TIT), compléments cognitifs, traduction littéraire, argent, Eugénie Grandet

\section{ABSTRACT}

The paper aims to emphasize that the use of cognitive complements during the translation process is essential. Based on the Interpretive Theory of Translation (ITT), this analysis sees translation as a cognitive approach. In the first instance, the author explores the presence of cognitive processes during the three phases of translation: comprehension, deverbalization, reformulation. Thereafter, the paper shows the difference between linguistic and extralinguistic elements that contribute to translation and stresses the importance of linguistic competencies and of the 
encyclopedic knowledge. Cognitive complements, understood as extralinguistic parameters, notional and affective, that contribute to understanding sense, help the translator carry the message not only from one language to another, but also from one cognitive world to another. In order to solve translation difficulties, the translator resorts to cognitive complements and adopts adequate strategies. When he/she wants to preserve the particularities of the source cognitive milieu, the translator uses literary translation or explicitations. On the contrary, when the translator intends to alleviate the specificities of the cognitive environment presented in the source text, he/she resorts to generalizing techniques or to adaptations. A case study is also included in the paper: the analysis of the way in which explicit and implicit references to money in an excerpt of the novel Eugenie Grandet by Balzac have been translated into English and Romanian. The analysis of the corpus shows that translation strategies should be chosen according to the knowledge that the translator shares with the target audience. Keywords: Interpretive Theory of Translation (ITT), cognitive complements, literary translation, money, Eugénie Grandet

\section{EXTENDED ABSTRACT}

The paper is based on the main principles of the Interpretive Theory of Translation (ITT) belonging to Danica Seleskovitch and Marianne Lederer. The two scholars established this approach as a result of their experience as conference interpreters. According to this theory, the translation process comprises three steps: understanding of sense, deverbalization of the message, and reformulation in the target language. During the first step, the translator seizes the message of the source text, then he/she "forgets" the linguistic form of the message, in order to find the best way to reformulate it. The ITT has an important impact in the field of Translation Studies, as it emphasizes the importance of translation seen rather as a process than as a product and the active role of the translator during this process. According to the ITT, translating is an interpretive action.

A successful translation renders the sense of the source text. In order to transpose the sense, the translator resorts to cognitive complements: they are notional and emotional parameters that help the translator understand, deverbalize and reformulate the sense in the target language. Extralinguistic knowledge is one such example. In the absence of cognitive complements, the translation becomes mere transcoding.

In our opinion, the impact of cognitive complements has been insufficiently developed in Translation Studies, even if they are essential to the accurate rendering of sense. In order to understand the role of cognitive complements in translation, one has to see translation as a cognitive process, a process that occurs in the mind of the translator. The purpose of this paper is, therefore, to emphasize the importance of cognitive complements during the three steps of the translation. 
In order to do so, we have selected a corpus that includes an excerpt of the novel Eugenie Grandet by Honoré de Balzac and its translation into English and Romanian. Using several examples retrieved from the corpus, we show that the use of cognitive complements is consistent during the three steps of translation. We analyze the role of linguistic hints and of extralinguistic knowledge in translation and stress the importance of cognitive complements throughout the entire process.

In the theoretical part, we operate a distinction between background information and the cognitive context: while the encyclopedic knowledge of the translator is essential to an accurate and legible rendering of sense, references to what has already been said in the source text are also essential, ensuring coherence. Memory plays a crucial role in this respect, as it helps the translator recover all the elements that are part of the cognitive context.

The theoretical analysis also shows that translating means not only carrying the meaning from one language to another, but also from one cognitive environment to another. In order to do so, the translator resorts to specific strategies. The particularities of the source cognitive milieu are preserved in the target language through literary translation and explicitation. On the other hand, the features of the source cognitive environment are leveled in translation through generalization and adaptation.

The leitmotiv of Eugenie Grandet is money: therefore, in the applicative part of the paper we analyze the way in which explicit and implicit references to money are translated into the target languages. In order to render sense in an adequate manner, the translator should consider the knowledge he/she shares with the target audience and should adapt his/her strategies accordingly. If cognitive complements are appropriately used, literary translation is avoided when it does not elucidate the sense. Also, the translator has to clarify implicit references when they are too cryptic for the target public.

As translation is a cognitive act, the translator and his/her manner of approaching the source text are of outmost importance. Cognitive complements are crucial in translation, as they contain the information needed in order to accurately comprehend, deverbalize and rephrase the message in the target language. Therefore, translating as a process and the role of cognitive complements should be more visible in the field of Translation Studies. 


\section{Introduction}

La traduction est un phénomène complexe, pratiqué depuis l'aube de l'histoire. Depuis la mythique «Tour de Babel», les traducteurs facilitent la communication entre les différentes langues du monde. Vrais médiateurs culturels, ils transportent le sens d'un idiome à l'autre - opération indispensable, qui comporte pourtant des risques, des compromis et des défaites.

La réflexion sur la traduction est, néanmoins, de date récente. La traductologie, telle qu'est dénommée dans le milieu francophone la théorie de la traduction, est née dans les années '60 avec les études de certains linguistes, tels que Georges Mounin, et, par la suite, des auteurs de la stylistique comparée (Jean-Paul Vinay et Jean Darbelnet au Canada). Plus tard, on a pu distinguer une approche philosophique de la traduction (représentée par Antoine Berman, Henri Meschonnic, Paul Ricoeur ou Jean-René Ladmiral.).

Si les théories de la traduction énumérées ci-dessus visent plutôt la traduction comme produit et comportent en général une visée prescriptive, la théorie interprétative de la traduction (TIT) conçue par Danica Seleskovitch et Marianne Lederer est née de la pratique d'interprétation de conférence et prend en compte avec priorité le traduire et les processus mentaux qui régissent les choix opérés par le sujet traduisant. Ce changement d'optique nous a semblé important et original. En outre, nous avons découvert que, tout comme la traduction générale ou spécialisée, la traduction littéraire peut faire l'objet de la théorie interprétative.

Plus précisément, la présente étude souligne l'importance des compléments cognitifs dans la traduction du discours littéraire. Fondée sur la théorie interprétative de la traduction, notre approche analyse la traduction en tant que processus cognitif, dont le succès dépend de différents paramètres, tels que le contexte et les compétences linguistiques et extralinguistiques que possède le traducteur. Selon nous, l'importance des compléments cognitifs, définis comme les paramètres extralinguistiques qui assurent la compréhension du sens, y compris les connaissances encyclopédiques du sujet traduisant, a été insuffisamment soulignée en traductologie.

Notre démarche suit les trois étapes de la traduction interprétative - la compréhension, la déverbalisation, la réexpression - pour voir à quel point la cognition est présente, 
pour distinguer par la suite les éléments linguistiques de l'extralinguistique et identifier le rôle des compléments cognitifs mobilisés par le sujet traduisant et les stratégies de traduction employées afin de «transporter » le sens dans la langue d'accueil. Pour illustrer notre propos, nous analysons dans la partie applicative du travail la traduction en anglais et en roumain de quelques références explicites et implicites à l'argent que I'on rencontre dans un fragment du roman Eugénie Grandet de Balzac. Le corpus comprend l'original en français, une traduction en anglais qui émane de Katharine Prescott Wormeley, réalisée à la fin du $19^{e}$ siècle, et une version en roumain dont l'auteur est Cezar Petrescu, parue en 1950 et rééditée au fil du temps.

\section{Le traduire comme démarche cognitive. La cognition et les étapes de la traduction selon la TIT}

Dans le domaine de la traductologie, la TIT est originale à plus d'un titre. II s'agit, premièrement, d'une théorie inspirée par l'expérience d'interprètes de conférence de ses auteures et non d'une simple démarche prescriptive. Ensuite, par le fait qu'elle identifie trois étapes du processus traductif (la compréhension ou saisie primaire du sens, la déverbalisation, qui comporte l'oubli de la forme linguistique du message et le déchiffrement du sens, et la réexpression ou reformulation du message dans la langue d'accueil), la TIT met l'accent plutôt sur le traduire que sur le produit fini, la traduction. Le traduire n'est abordé qu'au passage par les autres théories de la traduction, surtout parce qu'il est difficile de dire ce qui se passe vraiment dans le cerveau du sujet traduisant. Il y a pourtant des traductologues qui, ayant saisi l'importance des mécanismes qui régissent les choix traductifs, définissent le traduire comme une action plurielle, toujours autre devant chaque texte, effectuée par un «traduisant », qui s'inscrit dans un contexte culturel, social et historique. Dans la lignée de Meschonnic, le traduire est une « opération de lecture-écriture » (Vrinat-Nikolov \& Maurus, 2018, p. 119). Avec le traduire entre en scène le traducteur avec sa cognition, son style et ses préférences. II convient donc de souligner que la TIT met sur le devant de la scène le traduire en tant que processus cognitif et le sujet traduisant en tant qu'acteur.

De ce point de vue, force est de constater que la dimension cognitive n'a été que rarement traitée, et au passage, par les études de traductologie : « ll est intéressant de constater que la traductologie, discipline jeune en tant qu'activité scientifique et justiciable d'un enseignement universitaire, mais ancienne en tant que dispositif de réflexion, n'avait pas intégré dans sa réflexion jusqu'à il y a peu la dimension cognitive » 
(Balliu, 2007, p. 3). Jean-René Ladmiral, qui conteste la conceptualisation de la déverbalisation en tant qu'étape médiane de la traduction, distingue en effet ce qu'il appelle « la traductologie inductive », qui relèverait, selon lui, des neurosciences, de la traductologie " productive », et cela parce qu'« on ne sait pas encore ce qui se passe vraiment dans le cerveau du traducteur » $(2005$, p. 481). Selon le même auteur, la manière dont le traducteur déverbalise le message ne ferait pas l'objet de la traductologie, mais plutôt des sciences cognitives :

On se situe là sur un plan qui oscille entre le verbal et le protoverbal, le paraverbal et le non-verbal [...]. S'agissant d'une telle nébuleuse sémantique, on pourra en chercher des éléments d'analyse du côté de la linguistique cognitive [...]. Mais, au sein des sciences cognitives, c'est plus profondément du côté de la psycholinguistique qu'il y aura lieu d'attendre des éclaircissements sur le plan de la réalité objective du fonctionnement mental. (Ladmiral, 2005, p. 480)

Les processus mentaux qui se produisent dans le cerveau du sujet traduisant sont difficilement décelables, selon Ladmiral, et, de toute manière, ne constituent pas l'objet d'étude de la traductologie classique : «Cela dit, pour une traductologie inductive ou scientifique, l'essentiel regarde du côté de la psychologie cognitive, autant et plus que du côté de la linguistique » (2005, p. 483).

Néanmoins, la traduction n'est pas un simple transcodage, mais un processus mental, qui témoigne de la logique et du pouvoir de compréhension du traducteur, mais aussi de ses connaissances d'ordre linguistique et extralinguistique. Le fait qu'on ne sait pas pour l'instant avec exactitude comment fonctionne le cerveau du traducteur ne devrait pas éliminer ce sujet du domaine de la traductologie. De toute manière, les choix opérés par le traducteur au niveau du texte cible peuvent être parfois déduits par l'intermédiaire d'un processus que nous appellerions « déchiffrement inverse » : analyser la version finale pour déduire comment le traducteur a déverbalisé et compris le sens de départ, sens qui est défini par Lederer comme « l'idée ou si l'on préfère le vouloir dire du locuteur, et chez l'auditeur, le compris » (Seleskovitch \& Lederer, 1984, p. 256).

Pour démontrer nos propos, nous avons choisi deux traductions du roman Eugénie Grandet de Balzac : la première en anglais et la seconde en roumain. Le choix de l'œuvre source, vrai panorama d'une époque, est expliqué par la finesse du style de son auteur 
et le thème complexe et intéressant à analyser, y compris dans une perspective traductologique : I'argent. Paru en 1834, le roman, élaboré dans le style réaliste préféré par Balzac, décrit les différentes typologies présentes en France pendant la Restauration. L'accent est mis sur l'avarice et l'inflexibilité du père Grandet, qui contraste avec la naïveté de sa fille, Eugénie.

Le thème de l'argent est omniprésent dans le roman, étant évoqué directement ou par l'intermédiaire des allusions. Surtout en cas des références implicites, le sens n'est pas toujours facilement saisissable et traduisible. Soit l'exemple suivant extrait de notre corpus :

\section{Tableau 1 : Traduire les références implicites à l'argent}

\begin{tabular}{|c|c|c|}
\hline Texte source & Texte cible (en anglais) & Texte cible (en roumain) \\
\hline $\begin{array}{l}\text { Fifille, [...] au lieu de signer } \\
\text { cet acte qui coûtera gros à } \\
\text { faire enregistrer, si tu voulais } \\
\text { renoncer }[. . .] \text { à la succession } \\
\text { de ta pauvre chère mère } \\
\text { défunte, et t'en rapporter à moi } \\
\text { pour l'avenir, j'aimerais mieux } \\
\text { ça (1855, p. 315-316). }\end{array}$ & $\begin{array}{l}\text { My little girl [...], if, instead of } \\
\text { signing this deed, which will } \\
\text { cost a great deal to record, you } \\
\text { would [...] agree to renounce } \\
\text { your rights as heir to your } \\
\text { poor dear, deceased mother's } \\
\text { property, and would trust me } \\
\text { for the future, I should like it } \\
\text { better (1889, p. 250). }\end{array}$ & $\begin{array}{l}\text { Fetico, [...] în loc să iscălești } \\
\text { actul ăsta, a cărui înregistrare } \\
\text { ar costa scump, n-ar fi mai bine } \\
\text { să renunți [...] la moștenirea } \\
\text { răposatei tale mame și să-mi } \\
\text { treci mie tot ce ai? (2020, p. } \\
\text { 228). }\end{array}$ \\
\hline
\end{tabular}

Le fragment qui a attiré notre attention, marqué en italiques, est « t'en rapporter à moi pour l'avenir ». Le sens de l'expression «se rapporter à quelqu'un», dans un tel contexte, est, effectivement, celui de "faire confiance à quelqu'un ». La version en anglais reproduit fidèlement cette idée (" would trust me for the future »), sans être pourtant une traduction littérale. Par l'intermédiaire du déchiffrement inverse que nous avons évoqué ci-dessus, le critique de la traduction comprend que la traductrice a déverbalisé correctement l'expression française, offrant l'équivalent le plus proche en anglais. Quant à la version en roumain, «să-mi treci mie tot ce ai » (littéralement : « me céder en entier la fortune qui te revient »), elle fait preuve d'une infidélité manifeste. II s'agit en effet d'une surtraduction, parce que le texte d'arrivée dit plus que le texte de départ. Ce choix du traducteur anticipe en effet le déroulement de l'action romanesque et change la voix du texte, puisque le discours du père Grandet devient moins subtil. Par déchiffrement inverse on comprend donc que le traducteur s'est éloigné du sens de départ lors de l'étape de la déverbalisation, ce qui a changé complètement le message du texte balzacien. 
La traduction est, indéniablement, le produit des processus cognitifs qui ont lieu dans la tête du traducteur. Ne pas étudier la manière dont le message a été compris et déverbalisé pour être par la suite réexprimé dans la langue d'accueil exclut le sujet traduisant et sa manière d'aborder et de s'approprier le texte des recherches sur la traduction, ce qui met l'accent plutôt sur la traduction en tant que produit que sur le traduire. Or, le traduire est surtout une démarche cognitive.

La cognition comprend tous les processus mentaux relatifs à la compréhension et à l'acquisition de connaissances. La manière dont le message est compris dépend, premièrement, des connaissances linguistiques du traducteur, mais aussi, et parfois en égale mesure, de ses connaissances encyclopédiques, de ce que les théoriciennes de la TIT appellent « bagage cognitif ». Marianne Lederer ne sépare pas le cognitif de I'affectif : "Cognitif est utilisé comme adjectif de 'connaître' et comme substantif - le cognitif. Le cognitif est toujours simultanément affectif, aussi nous contentons-nous souvent du terme cognitif pour désigner le cognitif/affectif. Nous utilisons dans le même sens le couple notionnel/émotionnel. » (1994, p. 178) Comme le cognitif est intimement lié à l'action de comprendre, il va sans dire qu'il est présent dès la première étape du processus de traduction, lorsque le traducteur s'efforce de déchiffrer le sens. Par exemple, lorsqu'il/elle lit la phrase « Le lendemain de cette mort, Eugénie trouva de nouveaux motifs de s'attacher à cette maison où elle était née... » (p. 313), il/elle fera appel au contexte cognitif et comprendra qu'il s'agit du décès de la mère de l’héroïne.

Par la suite, la déverbalisation, qui suppose le détachement de la forme linguistique source et la saisie du sens brut, implique des processus cognitifs fins et précis. Dans la phrase citée ci-dessus, par exemple, le verbe "s'attacher » devra faire l'objet d'un déchiffrement minutieux : dans le contexte donné, il a un sens figuré et signifie plutôt « éprouver un sentiment durable pour quelque chose ». Le sujet traduisant laisse de côté l'enveloppe linguistique, à la recherche du sens.

Ladmiral, qui n'est pas d'accord avec la théorisation du concept de déverbalisation en traductologie, reconnait néanmoins que cette étape médiane existe, étant le résultat de la cognition :

Dans le cas de la traduction proprement dite [...], cette interphase est de nature psychologique ou, si l'on veut, 'mentale'. Plus précisément, [...] le message [...] passe du niveau verbo-linguistique à un niveau psycho- 
cognitif. C'est un point sur lequel on insiste à juste titre à l'É.S.I.T. en parlant aussi de conceptualisation pour qualifier ce moment inter-médiaire de la déverbalisation. (2005, p. 478)

Si la déverbalisation représente, probablement, l'apogée des processus cognitifs qui sont entrainés lors du traduire, confondre cette étape avec la cognition serait une erreur : «Effectivement, dans la Théorie interprétative de la traduction (Théorie du sens), la déverbalisation n'est que la deuxième des trois phases nécessaires à la médiation interlinguistique, alors que la cognition est mobilisée dans l'ensemble du processus » (Balliu, 2007, p. 3).

La réexpression est marquée à son tour par le cognitif. Lors de cette étape, le sujet traduisant cherche une forme sonore pour le sens qu'il vient de saisir. La solution offerte peut être plus ou moins réussie. Par exemple, le verbe "s'attacher (à cette maison) » ci-dessus est traduit quasi littéralement en anglais dans la version qui fait partie de notre corpus : " Eugenie felt a new motive for attachment to the house in which she was born... ». Le traducteur roumain, par contre, offre une solution maladroite parce qu'il fait recours au sens propre du verbe « s'attacher » : «Eugénie găsi mai puternice motive să se lege și mai sfâșietor de casa unde se născuse... » (littéralement : « ... de se lier à la maison où elle était née... »). On observe donc que « la réexpression mobilise l'ensemble de l'appareil cognitif du sujet. C'est un processus d'association du savoir linguistique et du savoir extralinguistique. La phase de réexpression est un mouvement non linéaire d'un vouloir-dire à sa formulation linguistique » (Djachy, 2013, p. 26).

Le travail de traduction entraine donc, dans son ensemble, des processus cognitifs indispensables au succès de la traduction. Dans ce cadre, il convient d'identifier clairement la différence entre le linguistique et l'extralinguistique en traduction, de souligner davantage l'importance des compléments cognitifs dont dispose le sujet traduisant et d'établir les stratégies à l'aide desquelles le sens peut être transféré dans une autre langue et culture qui supposent, en effet, une autre expérience collective cognitive.

\section{Le linguistique et l'extralinguistique. Les compléments cognitifs. Stratégies de traduction}

Conformément à la TIT, la tâche du sujet traduisant est de saisir le sens déverbalisé pour le reverbaliser par la suite dans la langue d'accueil. Pour ce faire, lors du travail interprétatif sont entrainées ses connaissances linguistiques et extralinguistiques : 
La réexpression de concepts d'une langue en une autre ne met pas en cause uniquement la faculté interprétative du traducteur, mais aussi ses connaissances. Pour bien comprendre le processus cognitif de la traduction, il ne faut pas confondre la démarche interprétative du sens du discours et les connaissances encyclopédiques et linguistiques sur lesquelles elle s'appuie. (Delisle, 1980, p. 112)

Selon nous, les disciples de la TIT ont souligné avec priorité l'importance de l'interprétation comme principe de base de l'acte traductif, tandis que le rôle des connaissances extralinguistiques est passé au second plan. Néanmoins, y compris dans la traduction littéraire, l'appel à l'encyclopédique est indispensable : « Le sens est compris par le traducteur (ou l'interprète) lorsqu'il ente des compléments cognitifs et affectifs sur un énoncé. La somme du linguistique et de l'extralinguistique confère donc à l'énoncé un sens, ce qui le distingue fondamentalement de la phrase, laquelle est une notion strictement linguistique. » (Balliu, 2007, p. 4) En outre, le transfert réussi du message dans la langue d'accueil dépend parfois fondamentalement de l'appel à l'extralinguistique : «La compréhension du sens par l'auditeur dépend même très largement de la présence ou de l'absence de ces engrammes non verbaux. Si celui-ci ne dispose pas des connaissances nécessaires, il ne pourra pas appréhender le vouloir dire de l'orateur et ne dépassera pas le stade de l'appréhension des significations linguistiques. » (Laplace, 1995, p. 211)

Le linguistique suppose, chez le sujet traduisant, comprendre le sens exprimé dans la langue source et le réexprimer selon les rigueurs de la langue cible, mais aussi réaliser des connexions entre le message à traduire et des éléments antérieurs du discours, qui sont activés grâce à la mémoire immédiate. À l'aide de cette mémoire, le traducteur se rendra compte que le syntagme " cette mort » du contexte "Le lendemain de cette mort, Eugénie trouva de nouveaux motifs de s'attacher à cette maison où elle était née... » (p. 313) fait référence au décès de la mère d'Eugénie évoqué auparavant dans le récit. Par contre, le terme « tonnelier » du syntagme « le vieux tonnelier » n'est pas si facile à traduire. Pour trouver un équivalent dans la langue d'accueil le sujet traduisant est tenu de faire appel à l'extralinguistique, à sa mémoire cognitive ou de réaliser une recherche dans le domaine. Katharine Prescott Wormeley omet ce terme dans sa version (" the old man »), peut-être parce qu'elle trouve qu'il est difficilement traduisible ou dépourvu d'importance dans le contexte donné, tandis que Cezar Petrescu le traduit littéralement (« bătrânul dogar »), sans expliciter, même si ce métier est obsolète à 
présent. Qu'il s'agisse d'éléments évoqués antérieurement dans le récit ou des connaissances extralinguistiques du sujet traduisant, le rôle de la mémoire est donc essentiel :

La mémoire joue un rôle principal dans le processus de compréhension. Ce processus fait intervenir une mémoire immédiate qui retient les mots pendant de brefs instants, ainsi qu'une mémoire cognitive qui contient l'ensemble des connaissances du sujet. Lors du processus de compréhension, la mémoire cognitive libère le savoir linguistique et extralinguistique pertinent. (Djachy, 2013, p. 25)

Soulignant l'importance du cognitif et des connaissances encyclopédiques dans la traduction, Christian Balliu appelle la mémoire liée à l'extralinguistique « mémoire déclarative » ou « sémantique » :

La mémoire sémantique rassemble les connaissances générales du monde, elle les organise sans tenir compte du moment ni des circonstances de leur constitution, dans le but de faciliter leur récupération : c'est la convocation, liée à une distribution en tiroirs. Cette distribution en fonction de la durée peut être regroupée dans ce qu'il est convenu d'appeler la mémoire déclarative (connaissances matérialisées dans le langage naturel ou par des images mentales). (2007, p. 8)

Le linguistique, activé par la mémoire immédiate, crée le contexte cognitif qui aide le sujet traduisant à réaliser des connexions avec des éléments antérieurs du discours ou du récit. Marianne Lederer voit dans le contexte cognitif un « savoir cumulatif » qui "se déverbalise mais reste présent en mémoire sous forme non verbale et aide le traducteur à comprendre son texte » (1994, p. 179). Par contre, Kétévan Djachy le voit comme « le stockage mnésique qui se constitue dans la mémoire depuis le début de la compréhension d'un texte » $(2013$, p. 24). L'extralinguistique, de l'autre côté, activé par la mémoire cognitive, fait référence au bagage cognitif que possède le traducteur. Le bagage cognitif est défini par Marianne Lederer comme « L'intégralité du savoir notionnel et émotionnel qu'un individu acquiert à travers 1 . son vécu personnel (savoir empirique), 2. le langage (ce qu'il apprend par la lecture, l'enseignement, les conversations, la télévision, etc.), 3. sa propre réflexion 4. et bien entendu sa connaissance d'une ou plusieurs langues » (1994, p. 178). À son tour, Kétévan Djachy considère que «le bagage 
cognitif se compose du savoir de l'individu contenu dans la mémoire d'une manière déverbalisée et est acquis à travers l'expérience personnelle, le langage et la réflexion » (2013, p. 24).

Sans appel au bagage cognitif, la traduction est vouée à l'échec et le résultat ainsi obtenu ressemble aux versions offertes par les moteurs de traduction automatique : "L'épaisseur de la compréhension d'un discours ou d'un texte dépend de l'étendue du bagage cognitif pertinent. En l'absence de recours au bagage cognitif, la traduction devient transcodage. » (Lederer, 1994, p. 178) L'acte interprétatif ne peut se produire, en effet, qu'en relation avec les éléments du bagage cognitif du sujet traduisant : « Pour donner vie aux signes couchés sur le papier, il importe non seulement de repérer les éléments pertinents mais aussi de les interpréter, comme c'est le cas dans le texte pragmatique, à la lumière d'un bagage cognitif préexistant » (Israël \& Lederer, 1991, p. 33).

La compréhension est donc impossible en l'absence des compléments cognitifs, définis par Marianne Lederer comme " des éléments pertinents, notionnels et émotionnels, du bagage cognitif et du contexte cognitif qui s'associent aux significations linguistiques des discours et des textes pour constituer le sens. Ils sont aussi indispensables à l'interprétation de la chaîne sonore ou graphique que la connaissance linguistique » (1994, p. 212). On observe par conséquent que les compléments cognitifs peuvent faire partie en égale mesure du contexte cognitif créé par le texte et du bagage cognitif du traducteur. Dans la terminologie de la TIT, les compléments cognitifs sont l'équivalent de l'extralinguistique, sans se limiter pourtant aux simples connaissances encyclopédiques du sujet traduisant. Dans l'ouvrage Terminologie de la traduction, les compléments cognitifs sont définis comme « des connaissances extralinguistiques mobilisées par le traducteur au moment où il cherche une équivalence et qui contribuent à la constitution du sens ». Néanmoins, le même travail précise que « les renseignements concernant l'auteur et les destinataires du texte, la connaissance du domaine et le contexte cognitif sont des compléments cognitifs » (Delisle \& Lee-Jahnke \& Cormier, 1999, p. 20). Le recours efficace aux compléments cognitifs facilite la compréhension du sens par la diminution de l'ambigu et l'éclaircissement de la polysémie :

Background knowledge is a blanket expression covering a number of cognitive complements that help us understand speech. These include knowledge of the world, of time and place, of the circumstances out of 
which speech arises, memory of things said previously, knowing who the speaker is and who the listeners are.

The broader the cognitive complements, the less ambiguity and polysemy there is in language, and the more thoroughly the speech is understood. (Lederer, 1990, p. 53)

Pour illustrer le rôle des compléments cognitifs dans la traduction littéraire, nous présentons ci-dessous une étude de cas. Soit le contexte suivant, extrait de notre corpus :

\section{Tableau 2 : Le rôle des compléments cognitifs dans la traduction littéraire}

\begin{tabular}{|c|c|c|}
\hline Texte source & Texte cible (en anglais) & Texte cible (en roumain) \\
\hline $\begin{array}{l}\text { [...] il faudrait signer cet acte } \\
\text { par lequel vous renonceriez } \\
\text { à la succession de madame } \\
\text { votre mère, et laisseriez à votre } \\
\text { père l'usufruit de tous les biens } \\
\text { indivis entre vous, et dont il } \\
\text { vous assure la nue-propriété... } \\
\text { (1855, p. } 315) \text {. }\end{array}$ & $\begin{array}{l}\text { [...] it is necessary to sign this } \\
\text { deed, by which you renounce } \\
\text { your rights to your mother's } \\
\text { estate and leave your father } \\
\text { the use and disposition, during } \\
\text { his lifetime, of all the property } \\
\text { undivided between you, of } \\
\text { which he guarantees you the } \\
\text { capital (1889, p. 250). }\end{array}$ & $\begin{array}{l}\text { Ar trebui să iscălești actul } \\
\text { acesta, prin care renunți } \\
\text { la moștenirea mamei și lași } \\
\text { uzufructul bunurilor aflate } \\
\text { in indiviziune, a căror nudă } \\
\text { proprietate și-o asigură (2020, } \\
\text { p. 227). }\end{array}$ \\
\hline
\end{tabular}

Pour transposer ce fragment, le sujet traduisant est tenu de faire recours au contexte cognitif et à ses connaissances encyclopédiques, en d'autres termes aux compléments cognitifs appropriés. La mémoire immédiate l'aidera à comprendre que le syntagme « cet acte » fait référence au document conclu par Maitre Cruchot à la demande du père Grandet par lequel le dernier se proposait de convaincre sa fille Eugénie de renoncer à la fortune qui lui revenait de la part de sa mère. L'équivalent anglais (« this deed ») est un terme à nuance juridique prononcée. Par contre, le traducteur roumain choisit la version littérale, qui n'est pas un terme spécialisé (« actul acesta »). En outre, le sujet traduisant fera appel au contexte cognitif, à ce que le texte lui a déjà révélé, et comprendra que l'expression « madame votre mère » employée par Cruchot fait référence à la mère d'Eugénie qui venait de mourir.

Néanmoins, la difficulté de traduction la plus manifeste est représentée dans ce contexte par les termes juridiques, qui parfois ne sont pas très familiers au traducteur littéraire. Dans ce cas, le sujet traduisant doit activer des éléments de son bagage 
cognitif ou faire une recherche dans le domaine pour se renseigner sur la terminologie. Heureusement, la traduction vers le roumain présente seulement la difficulté d'ordre terminologique : comme le droit roumain s'est amplement inspiré du droit français, le traducteur cherche la simple équivalence des termes et traduit littéralement «l'usufruit » par « uzufructul », « les biens indivis entre vous » par « bunurile aflate în indiviziune » et « la nue-propriété » par «nuda proprietate ». Seulement le terme « succession » est traduit par un terme plus général (« moștenirea » «l'héritage »), ce qui représente, en quelque sorte, un écart du registre juridique employé par Balzac. Par contre, I'analyse des mêmes termes en anglais frise l'intraduisible, parce que le droit anglo-saxon, fondé sur le précédent judiciaire et non sur la loi, est très différent du droit français. Par conséquent, beaucoup de termes du domaine des successions n'ont aucun équivalent dans la langue cible. Pour cette raison, la traductrice recourt souvent à des explicitations et traduit « la succession de madame votre mère » par " your rights to your mother's estate », « l'usufruit » par « the use and abuse, during his lifetime » et « il vous assure la nue-propriété » par « he guarantees you the capital ». Le syntagme « les biens indivis entre vous » est traduit par contre littéralement (« all the property undivided between you »), ce qui peut créer de la confusion pour les lecteurs américains, qui ne connaissent pas ce concept qui relève du droit français des successions.

On comprend donc que, sans l'appel aux compléments cognitifs, la traduction du fragment ci-dessus est quasi impossible. En d'autres termes, le contexte cognitif et les éléments du bagage cognitif du traducteur assurent la compréhension du sens par le lectorat. L'extralinguistique a un rôle tout aussi important que le linguistique, tel que le souligne Marianne Lederer : «In conversation, when we are listening to each other, the part played by knowledge of language can hardly be discerned from that played by background information. [...] Background knowledge is as important as command of language in understanding speech » (1990, p. 53).

La traduction s'avère être, en effet, un passage non seulement d'une langue à l'autre, mais aussi d'un univers cognitif à un autre univers cognitif, qui comporte parfois des éléments cognitifs différents, tel que le souligne Milena Srpová :

La traduction n'est pas seulement la confrontation de deux systèmes linguistiques face à une même réalité (une même culture, un même savoir cognitif), mais elle est aussi la confrontation de deux réalités (deux cultures, deux savoirs cognitifs stéréotypés). La traduction comme processus est 
donc non seulement un passage d'une langue à l'autre par le biais d'un savoir universel, mais aussi d'un univers extralinguistique à un autre, ou, pourrions-nous dire, d'une expérience collective cognitive à une autre expérience collective cognitive. (1995, p. 7)

Les difficultés de traduction les plus importantes apparaissent lorsque les deux univers cognitifs sont très éloignés et comportent plutôt des différences que des similitudes, comme dans le cas de la traduction en anglais analysée ci-dessus. Le sujet traduisant est tenu de prendre en compte les différences d'ordre linguistique et extralinguistique, c'est-à-dire " les écarts entre le savoir partagé dans la culture de départ et le savoir partagé dans la culture d'arrivée » (Srpová, 1995, p. 10). Il convient d'analyser quelles sont les options pour le sujet traduisant et quelles sont les stratégies de traduction les plus usitées pour surmonter ces difficultés qui relèvent du spécifique de chaque univers cognitif.

Les particularités de chaque univers cognitif peuvent être soit préservées, soit neutralisées lors du traduire. Dans l'étude de cas ci-dessus, le traducteur vers le roumain choisit plutôt de préserver les éléments de l'univers cognitif source, tandis que la traductrice vers l'anglais est obligée d'utiliser des paraphrases qui comportent des termes généralisants. Milena Srpová considère que «la conservation des spécificités cognitivo-référentielles [...] passe par la traduction littérale (et ses cas spécifiques l'emprunt et le calque), accompagnée ou non de l'explication du sens », stratégie qui, selon elle, était préférée à l'époque romantique, tandis que « la suppression des spécificités cognitivo-référentielles [...] aboutit à une traduction généralisante [...] ou à une traduction adaptative ", technique qui, selon elle, a été privilégiée à l'époque classique (1995, p. 11). Le choix de l'une ou de l'autre approche dépend du style de chaque traducteur/ traductrice, mais aussi du spécifique de la langue et de la culture d'arrivée, du contexte cible et, surtout, du savoir que le sujet traduisant partage avec le lectorat. De toute manière et quelle que soit l'approche adoptée, l'appel aux compléments cognitifs est indispensable pour faire passer le sens d'une langue à l'autre.

\section{Traduire l'argent - leitmotiv du roman Eugénie Grandet - à l'aide des compléments cognitifs}

Pour illustrer l'importance des compléments cognitifs dans la traduction littéraire, nous analyserons dans ce qui suit la traduction en anglais et, respectivement, en roumain 
d'un fragment représentatif du roman Eugénie Grandet de Balzac. II s'agit de la scène où, après la mort de sa femme, père Grandet parvient à convaincre sa fille de renoncer à la succession de sa mère (pages 313-317 de l'édition citée dans la bibliographie). Nous avons découpé des fragments qui évoquent le thème central du roman : I'argent. Tenant compte des principes de la TIT, nous avons partagé ces occurrences en deux classes : références explicites et, respectivement, implicites. En effet, c'est l'implicite qui, a piori, semble poser des problèmes plus importants au sujet traduisant. Les auteures de la TIT considèrent même que tout discours est hermétique :

Les discours sont toujours elliptiques, faits de langue en partie seulement, évoquant plus de cognitif qu'il n'en exprime. Chaque parole dit d'emblée plus qu'elle n'exprime; plus elle se déroule, plus l'explicite diminue au profit de l'implicite tandis que se crée chez l'auditeur une masse cognitive qui subsiste alors même que les mots qui l'ont matérialisée s'évanouissent. (Seleskovitch \& Lederer, 1984, p. 183)

Le sujet traduisant a donc une double tâche : déverbaliser l'implicite et, par la suite, le dévoiler ou non au lectorat cible en fonction du savoir partagé avec le dernier. Le but de l'analyse qui suit est de voir dans quelle mesure l'appel aux compléments cognitifs assure le passage adéquat du sens, qu'il s'agisse de références explicites ou implicites à l'argent.

\subsection{Traduire les références explicites à l'argent}

Même si l'argent est le thème central du roman Eugénie Grandet de Balzac, les références explicites qui y renvoient sont peu nombreuses, du moins dans le fragment qui fait l'objet de notre examen. On retrouve souvent, par exemple, des noms de monnaies : « livres » (p. 316), traduit par calque en anglais (« livres ») et littéralement en roumain (« livre »); «francs » (p. 317), rendu en anglais toujours par un calque («francs ») et en roumain par traduction littérale (« franci »). Le terme populaire « sou » (p. 317) est copié en tant que tel en anglais (« one sou »), ce qui sans doute rend la compréhension difficile. Par contre, le traducteur roumain choisit un terme argotique du même registre pour réexprimer le message (« o para »), ce qui représente un choix inspiré. Le terme « argent » (p. 314) est retrouvé une seule fois, dans le syntagme « argent comptant », traduit en anglais de manière discutable par « ready money » au lieu de « cash » et en roumain par l'équivalent fonctionnel (« bani lichizi »). Le terme 
«fortune » (p. 314) est transposé littéralement en anglais (« fortune ») et en roumain (« avere »). Le terme « biens » est à retrouver aussi dans le texte (p. 315), il étant omis dans la version en anglais et traduit littéralement en roumain (« bunurile»). Une référence explicite à l'argent est représentée par l'expression « une bonne grosse rente de cent francs » (p. 316). Le terme « rente » est interprété comme « montant » par la traductrice vers l'anglais, dont la version garde pourtant l'explicite ( " the good round sum of a hundred francs »). Le traducteur roumain, par contre, choisit une version trop littérale («frumoasa rentă de o sută de franci »), ce qui représente une solution inappropriée, parce qu'en roumain « rentă » signifie plutôt « loyer à payer ».

Dans le fragment qui fait l'objet de notre analyse on retrouve aussi de nombreux verbes qui renvoient directement au thème de l'argent. Un tel verbe est « coûter » (p. 315), traduit littéralement par « to cost » en anglais et « a costa » en roumain. La traduction littérale est en effet privilégiée pour rendre dans la langue d'arrivée les références directes à l'argent, tel que le montre le contexte ci-dessous :

\begin{tabular}{|c|c|c|}
\hline Texte source & Texte cible (en anglais) & Texte cible (en roumain) \\
\hline $\begin{array}{l}\text { Mademoiselle, monsieur votre } \\
\text { père ne voudrait ni partager, ni } \\
\text { vendre ses biens, ni payer des } \\
\text { droits énormes pour l'argent } \\
\text { comptant qu'il peut posséder. } \\
(1855, \text { p. } 314)\end{array}$ & $\begin{array}{l}\text { Mademoiselle, your father } \\
\text { does not wish to divide the } \\
\text { property, nor sell the estate, } \\
\text { nor pay enormous taxes on the } \\
\text { ready money which he may } \\
\text { possess. }(1889, \text { p. } 249)\end{array}$ & $\begin{array}{l}\text { Domnișoară, tatăl dumitale } \\
\text { n-ar voi nici să împartă, } \\
\text { nici să vândă bunurile sale, } \\
\text { nici să plătească sume cu } \\
\text { totul enorme față de puținii } \\
\text { bani lichizi pe care îi are la } \\
\text { îndemână. (2020, p. 227) }\end{array}$ \\
\hline
\end{tabular}

La traduction littérale est privilégiée par les deux traducteurs pour rendre ces verbes dans la langue cible. On observe également que le terme « droits » est correctement interprété comme « taxes » en anglais et « sume » (« montants ») en roumain. Le verbe « payer » est présent aussi dans le contexte ci-dessous :

\begin{tabular}{|l|l|l|}
\hline \multicolumn{4}{|l|}{ Tableau 4 : Traduire le verbe « payer » } \\
\hline Texte source & Texte cible (en anglais) & Texte cible (en roumain) \\
\hline $\begin{array}{l}\text { Vois, tu pourras payer autant } \\
\text { de messes que tu voudrais à } \\
\text { ceux pour lesquels tu en fais } \\
\text { dire... (1855, p. } 316)\end{array}$ & $\begin{array}{l}\text { many masses as you want for } \\
\text { anybody... }(1889, \text { p. } 250)\end{array}$ & $\begin{array}{l}\text { Vei putea plăti câte acatiste și } \\
\text { pomeniri vei pofti, de sufletul } \\
\text { morților tăi... (2020, p. 228) }\end{array}$ \\
\hline
\end{tabular}


Le verbe « payer » est littéralement rendu dans les deux langues d'accueil. Par contre, l'expression " payer des messes », qui est un culturème religieux, est transposée en tant que telle en anglais (« to pay for masses »), solution discutable d'ailleurs, et est adaptée par naturalisation en roumain (« a plăti acatiste și pomeniri »), tenant compte du spécifique du culte orthodoxe, le plus familier dans la culture cible.

L'analyse de la traduction des références directes à l'argent montre que la stratégie littérale est privilégiée pour transporter le sens dans la langue cible et cela peut-être parce que de telles références supposent un exercice minimal d'interprétation et l'équivalent est, en général, facilement identifiable. Pour trouver l'équivalent approprié, les traducteurs ont fait néanmoins appel aux compléments cognitifs, comme dans le cas de la traduction des termes juridiques analysés ci-dessus. Dans ce qui suit, nous examinerons les stratégies adoptées pour traduire en anglais et en roumain les références implicites à l'argent.

\subsection{Traduire les références implicites à l'argent}

Tout discours comporte de l'explicite et de l'implicite dont les réglages sont très fins : « [...] tout texte est un compromis entre un explicite suffisamment court pour ne pas laisser par l'énoncé de choses sues et un implicite suffisamment évident pour ne pas laisser le lecteur dans l'ignorance du sens désigné par l'explicite » (Lederer, 1994, p. 58). À la différence de l'explicite, traduire l'implicite comporte un exercice de déchiffrement et un effort plus important de reverbalisation, car le sujet traduisant doit décider de garder l'implicite en tant que tel ou de l'expliciter si cela est au profit du lectorat. En outre, l'implicite discursif oblige le traducteur/la traductrice de faire appel aux compléments cognitifs. Soit l'exemple suivant :

\section{Tableau 5 : Traduire l'implicite discursif}

\begin{tabular}{|l|l|l|}
\hline Texte source & Texte cible (en anglais) & Texte cible (en roumain) \\
\hline $\begin{array}{l}\text { [...] il la couvait comme si elle } \\
\text { eût été d'or. (1855, p. 313) }\end{array}$ & $\begin{array}{l}\text { [...] he brooded over her as } \\
\text { though she had been gold. } \\
(1889, \text { p. 248) }\end{array}$ & $\begin{array}{l}\text { [...] o sorbea cu ochii, parc-ar fi } \\
\text { fost de aur. (2020, p. 226) }\end{array}$ \\
\hline
\end{tabular}

La comparaison du texte source contient un renvoi subtil à l'argent : le substantif « l'or ». Pendant la réexpression, le sujet traduisant est censé trouver dans la langue cible une expression qui crée le même effet : heureusement, la traduction littérale est 
acceptable en anglais et en roumain. C'est au lecteur que revient la tâche d'identifier la référence implicite à la fortune, qui $a$, dans ce contexte, une valeur ironique : de manière inhabituelle, Grandet prend soin de sa fille et la regarde « d'un œil presque bon » (p. 313) parce qu'il a l'intérêt de le faire. L'implicite reste, donc, cryptique.

Un verbe qui fait référence implicitement à l'argent est « dépouiller », à retrouver deux fois dans le fragment qui fait l'objet de notre étude :

\begin{tabular}{|l|l|l|}
\hline \multicolumn{4}{|l|}{ Tableau 6 : Traduire le verbe « dépouiller » } \\
\hline Texte source & Texte cible (en anglais) & Texte cible (en roumain) \\
\hline $\begin{array}{l}\text { Ni vous ni ma fille ne voulez } \\
\text { me dépouiller. (1855, p. 315) }\end{array}$ & $\begin{array}{l}\text { Neither you nor my daughter } \\
\text { wish to rob me [...]. (1889, p. } \\
249)\end{array}$ & $\begin{array}{l}\text { Nici dumneata, nici fiica mea } \\
\text { nu voiți să mă despuiați. (2020, } \\
\text { p. 227) }\end{array}$ \\
\hline $\begin{array}{l}\text { Mademoiselle, [...] il est de } \\
\text { mon devoir de vous faire } \\
\text { observer que vous vous } \\
\text { dépouillez... (1855, p. 316) }\end{array}$ & $\begin{array}{l}\text { Mademoiselle, [...] it is my } \\
\text { duty to point out to you that } \\
\text { you are despoiling yourself } \\
\text { without guarantee [...]. (1889, } \\
\text { p. 250) }\end{array}$ & $\begin{array}{l}\text { Domnișoară, [...] sunt dator să } \\
\text { despuiați de avere... (2020, p. } \\
228)\end{array}$ \\
\hline
\end{tabular}

En français, le verbe « dépouiller » fait référence à l'action de déposséder quelqu'un, de le/la priver de quelque chose, de soustraire des biens à quelqu'un. Katharine Prescott Wormeley le traduit en anglais par « to rob » (« voler ») dans le premier contexte, ce qui représente, selon nous, un écart sémantique, s'agissant d'une action tout différente, moins subtile. Dans le deuxième cas, la traductrice choisit une paraphrase explicative qui garde l'équivalent littéral du verbe source (« to despoil oneself without guarantee »). Par contre, l'emploi du même équivalent littéral en roumain (" a despuia pe cineva », « a se despuia de avere ») est maladroit, car le verbe roumain n'a pas le sens du français, mais il signifie « déshabiller quelqu'un », ce qui crée de la confusion.

En étroite liaison avec le verbe « dépouiller » est le nom « spoliation » présent dans le contexte suivant :

\section{Tableau 7 : Traduire le nom « spoliation »}

\begin{tabular}{|l|l|l|}
\hline Texte source & Texte cible (en anglais) & Texte cible (en roumain) \\
\hline Le lendemain, vers midi, fut & The next morning Eugenie & A doua zi, către amiază, a fost \\
signée la déclaration par laquelle & $\begin{array}{l}\text { signed the papers by which } \\
\text { astfel iscălită declarația, prin } \\
\text { Eugénie accomplissait elle- } \\
\text { même sa spoliation. (1855, p. 317) }\end{array}$ & $\begin{array}{l}\text { spoliation. (1889, p. 251) } \\
\text { sare Eugénie se jefuia cu mâna } \\
\text { ei. (2020, p. 229) }\end{array}$ \\
\hline
\end{tabular}


Le terme est rendu littéralement en anglais ("spoliation ») et en utilisant une paraphrase explicative en roumain (« a se jefui cu mâna proprie » - « se dépouiller soimême »), ce qui représente une adaptation.

Le terme « affaire », utilisé à maintes reprises dans le texte, représente une référence implicite à l'argent. Il est interprété différemment par les deux traducteurs :

\begin{tabular}{|l|l|l|}
\hline \multicolumn{4}{|l|}{ Tableau 8 : Traduire le nom « affaire » } \\
\hline Texte source & Texte cible (en anglais) & Texte cible (en roumain) \\
\hline $\begin{array}{l}\text { [...] te voilà héritière de ta } \\
\text { mère, et nous avons de petites } \\
\text { affaires à régler entre nous } \\
\text { deux. (1855, p. 314) }\end{array}$ & $\begin{array}{l}\text { heiress, and we have few little } \\
\text { matters to settle between us. } \\
(1889, \text { p. } 249)\end{array}$ & $\begin{array}{l}{[\ldots] \text { iată-te moștenitoarea }} \\
\text { bietei tale mame; avem de pus } \\
\text { la punct unele lucruri. (2020, } \\
\text { pp. 226-227) }\end{array}$ \\
\hline $\begin{array}{l}\text { [...] nous sommes quittes. } \\
\begin{array}{l}\text { Voilà comment doivent se } \\
\text { faire les affaires. La vie est une } \\
\text { affaire. (1855, p. 316-317) }\end{array}\end{array}$ & $\begin{array}{l}\text { business should be done. Life is } \\
\text { a business. (1889, p. 251) }\end{array}$ & $\begin{array}{l}\text { [...] suntem chit! lată cum se fac } \\
\text { afacerile! (?) (2020, p. 228) }\end{array}$ \\
\hline
\end{tabular}

Le terme « affaires » du premier contexte est traduit par adaptation dans les deux langues cible (« matters » en anglais et, respectivement, « lucruri » (« choses ») en roumain), ce qui change le registre du discours. Par contre, dans le deuxième cas cité, le même terme est traduit littéralement (« business » en anglais et « afaceri » (« affaires ») en roumain). Le deuxième traducteur commet une omission qui porte atteinte grièvement au sens transmis : la phrase "La vie est une affaire », phrase-clé du roman de Balzac, n'est pas traduite en roumain.

L'implicite est rendu par traduction littérale lorsque le traducteur ne ressent pas le besoin de le rendre explicite, en d'autres termes lorsque le savoir qu'il partage avec le lecteur est quasi-identique. Par contre, lorsque l'implicite risque de rester hermétique aux yeux du public cible, les traducteurs adoptent des techniques d'explicitation ou d'adaptation aux rigueurs de la langue d'accueil. Dans les deux cas, les compléments cognitifs sont essentiels : « Cette relation entre l'implicite et l'explicite est donc une caractéristique essentielle du fonctionnement de la communication dans laquelle les compléments cognitifs du récepteur jouent un rôle fondamental »(Djachy, 2013, p. 25). 


\section{Conclusion}

L'analyse que nous avons menée dans le cadre du présent travail a mis en évidence le rôle de la cognition et des connaissances détenues par le sujet traduisant. L'appel aux compléments cognitifs a lieu pendant les trois étapes de la traduction - la compréhension, la déverbalisation, la réexpression - et assure le transport du sens dans la langue d'arrivée :

[...] le processus de traduction est conçu comme la compréhension du sens exprimé dans le texte de départ, formulé dans une langue de départ, et la réexpression de ce "même » sens dans le texte d'arrivée, formulé dans une langue d'arrivée. La compréhension (l'interprétation) du sens se fait par l'adjonction du savoir qu'a le traducteur des réalités et des objets désignés. Implicitement, cette conception repose sur le présupposé d'une réalité universelle. (Srpová, 1995, p. 7)

La traduction devient, donc, passage d'une réalité cognitive à une autre réalité cognitive. Au niveau du texte cible, le sujet traduisant met en œuvre des stratégies appropriées pour préserver ou niveler les spécificités cognitives et référentielles en fonction du savoir qu'il partage avec le lecteur. L'étude du corpus nous a montré que, lorsque le traducteur/la traductrice ne réexprime pas le message en fonction des connaissances que détient le lectorat, la traduction est soit trop explicative, soit trop hermétique. Le sujet traduisant doit trouver donc l'équilibre juste quand il s'agit de réexprimer l'explicite ou l'implicite dans la langue d'accueil.

De toute manière, sans recours aux compléments cognitifs, la traduction n'est plus un processus interprétatif, mais devient un simple transcodage, un mot-à-mot :

Since languages differ in all respects, not only in sound structures, semantics or syntax, but also in the way speakers refer to ideas, facts and events, interpreters cannot proceed directly from one language to the other. The only way for them to express the original information correctly is to resort to non-linguistic knowledge. Only cognitive complements can explain fully the nature of interpreting and vindicate the interpreter's assertion that understanding speech goes further than understanding language. (Lederer, 1990, p. 59) 
Comme l'analyse du corpus l'a montré, le recours aux compléments cognitifs est impératif dans la traduction écrite, y compris dans la traduction littéraire. De ce point de vue, le concept d'« interprète » évoqué par Marianne Lederer ci-dessus peut être compris comme une métaphore pour tout sujet qui traduit le sens, à l'oral ou à l'écrit.

Évaluation : Évaluation anonyme par des pairs extérieurs.

Conflit d'intérêts : L'auteure n'a aucun conflit d'intérêts à déclarer.

Subvention : L'auteure n'a reçu aucun soutien financier pour ce travail.

Peer-review: Externally peer-reviewed.

Conflict of Interest: The author has no conflict of interest to declare.

Grant Support: The author declared that this study has received no financial support.

\section{Bibliographie}

Balliu, C. (2007). Cognition et déverbalisation. Meta, 52(1), 3-12. https://doi.org/10.7202/014714ar.

Balzac, H. de (1855). Eugénie Grandet. Paris : Alexandre Houssiaux, Éditeur.

Balzac, H. de (1889). Eugenie Grandet. Translated by Katharine Prescott Wormeley. Boston: Roberts Brothers.

Balzac, H. de (2020). Eugénie Grandet. Traducere de Cezar Petrescu. Bucureşti : Litera.

Delisle J. (1980). L'analyse du discours comme méthode de traduction. Initiation à la traduction française de textes pragmatiques anglais. Théorie et pratique. Collection "Cahiers de traductologie », 2. Ottawa : Presses universitaires d'Ottawa.

Delisle, J \& Lee-Jahnke, H. \& Cormier M. (1999). Terminologie de la traduction. Amsterdam : John Benjamins.

Djachy, K. (2013). Le rôle de la TIT en traductologie. Le Linguiste. Revue Internationale en science du langage, no. 4,

Qom, pp. 21-30.

Israël, F. et Lederer M. (1991). La liberté en traduction. Paris : Didier Érudition.

Ladmiral, J.-R. (2005). Le " salto mortale de la déverbalisation ». Meta, 50(2), pp. 473-487. https://doi. org/10.7202/010994ar.

Laplace, C. (1994). Théorie du langage et théorie de la traduction : Les concepts clefs de trois auteurs, Kade (Leipzig),

Coseriu (Tübingen), Seleskovitch (Paris). Paris : Didier Erudition.

Lederer, M. (1990). The Role of Cognitive Complements in Interpreting. Interpreting - Yesterday, Today and

Tomorrow. American Translators Association. Scholarly Monograph Series, volume IV, edited by David and Margareta Bowen. State University of New York at Binghamton (SUNY), pp. 53-60.

Lederer, M. (1994). La Traduction aujourd'hui. Le modèle interprétatif. Paris : Hachette.

Seleskovitch, D. \& Lederer, M. (1984). Interpréter pour traduire. Paris : Didier Érudition.

Srpová, M. (1995). La traduction, confrontation de deux expériences cognitives. Intellectica, no. 1, volume 20, pp. 157-170. Vrinat-Nikolov, M. \& Maurus, P. (2018). Shakespeare a mal aux dents. Paris : Presses Inalco. 